Check for updates

Cite this: Mater. Adv., 2021, 2,6620

Received 18th May 2021,

Accepted 31st August 2021

DOI: $10.1039 / \mathrm{d} 1 \mathrm{ma} 00444 \mathrm{a}$

rsc.li/materials-advances

\section{Plasma assisted design of biocompatible 3D printed PCL/silver nanoparticle scaffolds: in vitro and in vivo analyses $\dagger$}

\author{
Neethu Ninan, ${ }^{\mathrm{ab}}$ Blessy Joseph, ${ }^{\mathrm{c}}$ Rahul Madathiparambil Visalakshan, ${ }^{\mathrm{ab}}$ \\ Richard Bright, ${ }^{\text {ab }}$ Clement Denoual, ${ }^{d}$ Peter Zilm, ${ }^{\text {P }}$ Yogesh Bharat Dalvi, ${ }^{f}$ P. V. Priya, ${ }^{9}$ \\ Aji Mathew, (D) ${ }^{\text {h }}$ Yves Grohens, ${ }^{d}$ Nandakumar Kalarikkal, ${ }^{c}$ Krasimir Vasilev (D) ${ }^{a b}$ and \\ Sabu Thomas (D) *ci
}

\begin{abstract}
3D printing provides numerous opportunities for designing tissue engineering constructs with intricate porosity, geometry and favourable mechanical properties and has the potential to revolutionize medical treatments. However, an often-encountered restriction is the selection of materials suitable for utilization in 3D printing, not all of which have appropriate biocompatibility properties. In this work, fused deposition modeling was employed to fabricate 3D PCL constructs without the use of any solvent. Plasma deposition was used to modify the surface of the scaffolds, followed by immobilization of silver nanoparticles. The physico-chemical and mechanical analyses demonstrated that the scaffolds retained their porosity and mechanical integrity. The mechanical properties evaluated by the nanoindentation technique demonstrated an increase in reduced modulus to $1.87 \pm 0.012 \mathrm{GPa}$ for $\mathrm{PCL}$ scaffolds functionalized with silver nanoparticles for 24 hours. We also showed complete prevention of colonization by medically relevant pathogens. The modified scaffolds had good biocompatibility. The immune response studies in the culture of macrophages confirmed a reduction in the level of expression of pro-inflammatory cytokines which is a key requirement for successful wound healing. The in vivo studies on Sprague Dawley rats indicated enhanced angiogenesis and the absence of foreign body reaction for scaffolds functionalized with silver nanoparticles for 6 hours. The 3D printing approach presented in this study provides new sustainable opportunities that can be adopted for designing biomaterial constructs with enhanced biological properties.
\end{abstract}

${ }^{a}$ Academic Unit of STEM, University of South Australia, Mawson Lakes, SA 5095, Australia. E-mail: neethun.ninan@gmail.com, krasimir.vasilev@unisa.edu.au

${ }^{b}$ Future Industries Institute, University of South Australia, Mawson Lakes, SA 5095, Australia

${ }^{c}$ International and Inter University Centre for Nanoscience and Nanotechnology, Mahatma Gandhi University, Kottayam 686 560, Kerala, India.

E-mail: sabuthomas@mgu.ac.in

${ }^{d}$ University Bretagne Sud, UMR CNRS 6027, IRDL, F-56100, Lorient, France

${ }^{e}$ Adelaide Dental School, University of Adelaide, South Australia, Australia

${ }^{f}$ Pushpagiri Research Centre, Thiruvalla 689101, Kerala, India

${ }^{g}$ Pathology Department, Government Medical College, Kottayam 686008, Kerala, India

${ }^{h}$ Division of Materials and Environmental Chemistry, Stockholm University, Frescativägen 8, 10691, Stockholm, Sweden

${ }^{i}$ School of Energy Studies and School of Chemical Sciences, Mahatma Gandhi University, Kottayam 686 560, Kerala, India

$\dagger$ Electronic supplementary information (ESI) available. See DOI: 10.1039/ d1ma00444a

\section{Introduction}

Extracellular matrix (ECM) is a 3-dimensional (3D) network with hierarchical topographical features, providing support and aiding cellular adhesion, differentiation and migration. ${ }^{1}$ ECM is a non-cellular component present in all tissues which when disrupted can lead to inflammatory responses that aggravate the wound healing process. ${ }^{2,3}$ To address the necessity for a better ECM substitute, several biofabrication techniques have evolved in the past decade. ${ }^{4-6}$ The goal is to generate tissue engineering scaffolds that create a microenvironment which resembles natural ECM and promote tissue reconstruction and replacement. Among the various biofabrication techniques, 3D printing is a technology with high process versatility and flexibility which permits spatiotemporal control of the communication between cells and ECM. ${ }^{7}$ It is a bottom-up approach that consists of bio-ink (polymers or cell-laden hydrogels) deposition in a layer-by-layer fashion to produce scaffolds with intricate porosity and geometry. ${ }^{8}$ The technique carries great 
promise in tissue modeling, pharmaceutics, replacing complex anatomical structures, tissue regeneration and other areas. ${ }^{9}$

Several synthetic polymers can be used in 3D printing because of their photochemical and thermal processability. However, their poor bioactivity leads to reduced cell anchorage and unfavourable protein adsorption. ${ }^{10}$ Polycaprolactone (PCL) is one such synthetic polymer approved by the Food and Drug Administration (FDA) for biomedical applications. ${ }^{11,12}$ It is an ideal material for $3 \mathrm{D}$ printing due to its low melting point. Nonetheless, the low surface energy and hydrophobicity of PCL make the polymer unfavourable for cell adhesion, leading to reduced biological performance. ${ }^{13-16}$ Attempts have been made to modify the surface of PCL using alkaline hydrolysis which often had a negative impact on material bulk properties and altered its morphology. ${ }^{17}$ The surface modification of PCL using polyethylene glycol has resulted in the production of oxides and free radicals that could impact the polarity of the surface. ${ }^{18}$ Application of functional coatings to PCL is an approach to mitigate these problems. Surface functionalization can be carried out via a physical or chemical attachment of ligands or biomolecules. ${ }^{19,20}$ The disadvantage of physical adsorption is the possibility of desorption from the surface. On the other hand, covalent immobilization overcomes the fundamental problems of instability and leaching of bioactive motifs attached to the surface. However, the chemistry underlying this immobilizing technique is often substratedependent, complex, multistep and involves the use of a large amount of solvent. ${ }^{21}$

Plasma polymerization is a substrate independent technology used to deposit a nano-thin layer of polymer on any type of material providing targeted and versatile surface engineering without changing bulk properties. ${ }^{22-25}$ In contrast, other techniques for thin layer fabrication such as layer-by-layer and selfassembled monolayers require a specific substrate. ${ }^{26}$ Thin layers deposited by plasma polymerization can be used to introduce reactive functional groups onto biomaterial surfaces that can effectively bind bioactive molecules and nanoparticles. ${ }^{27-29}$ The unique features of this facile method are the absence of solvents or initiators, minimal requirement of precursors and lack of liquid organic waste, making it environmentally friendly. ${ }^{23}$ Plasma technology has been already demonstrated to be a useful utility to modify 3D printed scaffolds. For example, acrylic acid-coated 3D printed PCL has resulted in enhanced growth of osteoblasts and mesenchymal stem cells. ${ }^{30}$

An issue with all medical devices and constructs, including those generated via 3D printing, is colonization by bacteria and subsequent infections. ${ }^{31,32}$ Infections with medical devices can be devastating to patients causing mortality and morbidity and add substantial costs to the healthcare systems worldwide. ${ }^{33,34}$ AgNPs are now well accepted for their antibacterial properties and have been applied to a range of medical devices. ${ }^{35,36}$ AgNPs are mixed with synthetic polymers or biopolymers to yield scaffolds with antibacterial properties. PCL wound dressings impregnated with ionic silver, zinc oxide and copper exhibited antibacterial properties. ${ }^{37}$ However, the study did not provide any evidence on their impact on biocompatibility or the immune response. PCL/AgNP composite scaffolds prepared by electrospinning and solvent casting displayed antibacterial activity but exhibited poor control over pore size and architecture compared to the 3D printing technique. ${ }^{38-40}$ Very recently, Radhakrishnan et al. reported 3D printing of PCL/AgNPs using fused deposition modeling. ${ }^{41}$ Here, PCL/AgNP scaffolds were fabricated using tetrahydrofuran and dimethylformamide as solvents which was further extruded. The main goal of our work was to avoid toxic solvents and maintain a sustainable approach. Gao et al. have prepared PCL nanocomposites containing octahedral AgNPs for bone tissue engineering. ${ }^{42} \mathrm{Ho}$ et al. have synthesised PCL/carbon nanotube nanocomposites for cardiac tissue engineering. ${ }^{43}$ Both these articles focus on nanocomposites where the bulk properties of PCL are significantly changed unlike the plasma surface engineering technique employed in this work.

Plasma methods were integrated with traditional tools of nanotechnology to confer antibacterial properties to PCL 3D printed scaffolds without compromising their mechanical integrity. To achieve that, we applied plasma polymer coatings engineered with silver nanoparticles (AgNPs) to the surface of $3 \mathrm{D}$ porous scaffolds. We hypothesized that the potent antimicrobial properties of AgNPs can be transferred to 3D printed constructs and this will make them resistant to bacterial colonization. However, a challenge remains to preserve the biocompatibility of the material after the application of an antibacterial coating and prevent cells and tissue cytotoxicity. Thus, in this study, we not only demonstrate the high antibacterial efficacy of the surface modified scaffolds, but also evaluate whether there may be any negative impact on the viability of primary human fibroblast cells. Furthermore, the evaluation of the nano-mechanical attributes of immobilized silver nanoparticles would provide an insight into the biomechanical properties of modified scaffolds. Important for applications, we also studied the innate immune responses of the new material in a culture of macrophages and evaluated their performance in vivo on male Sprague Dawley rats.

\section{Experimental}

\section{Materials}

PCL Capa ${ }^{\text {тм }} 6800$ (molecular weight $=80000 \mathrm{~g} \mathrm{~mol}^{-1}$ ) was purchased from Perstorp. Allyl amine (98\%), 2-mercaptosuccinic acid, sodium borohydride $\left(\mathrm{NaBH}_{4}\right)$, phosphatebuffered saline (PBS), Dulbecco's Modified Eagle's Medium (DMEM), and Rosewell Park Memorial Institute (RPMI) medium were purchased from Sigma-Aldrich. Silver nitrate was procured from Pro Sci-Tech. Penicillin and streptomycin were bought from Life Technologies. Fetal bovine serum (FBS) was obtained from Thermo Scientific. Tryptone Soy Broth (TSB) was purchased from Oxoid. For all experiments, ultra-pure Milli-Q water was used. Staphylococcus epidermidis (ATCC 35984) and Pseudomonas aeruginosa (NCTC 6749) were chosen for 
anti-bacterial experiments based on their ability to cause chronic wound infections.

Primary human foreskin fibroblasts (HFFF2) and monocyte cell lines (THP-1) were used in the experiments. HFFF2 were grown in DMEM consisting of 5\% heat inactivated FBS and 1\% $(\mathrm{v} / \mathrm{v})$ penicillin/streptomycin in a $5 \% \mathrm{CO}_{2}$ incubator maintained at $37{ }^{\circ} \mathrm{C}$. THP-1 was incubated in RPMI containing $5 \%$ heatinactivated FBS and $1 \%(\mathrm{v} / \mathrm{v})$ penicillin/streptomycin at $37{ }^{\circ} \mathrm{C}$ in a humidified atmosphere at $5 \% \mathrm{CO}_{2}$.

Ketamine Hydrochloride Injection (Aneket ${ }^{\circledR}$ ) and Xylazine Injection (Xylaxin ${ }^{\circledR}$ ) were used for the in vivo studies.

\section{Methods}

Fabrication of scaffolds via 3D printing. 3D printed scaffolds were fabricated using a MakerBot Replicator 2X 3D printer with a $0.4 \mathrm{~mm}$ diameter nozzle. Before extrusion, PCL pellets were dried in a vacuum oven set at $45{ }^{\circ} \mathrm{C}$ for $24 \mathrm{~h}$. The dried pellets were extruded using a SCAMEX single-screw extruder with a screw length/diameter $(L / D)$ ratio of 20 . The diameter of the cylindrical die was $2 \mathrm{~mm}$, and the filament thickness was adjusted to $1.75 \mathrm{~mm}$. The extruder was set at a temperature of $80{ }^{\circ} \mathrm{C}$ and the printing bed was at room temperature $\left(\sim 25{ }^{\circ} \mathrm{C}\right)$. The velocity of printing was $12.5 \mathrm{~mm} \mathrm{~s}^{-1}$ for the first layer and $25 \mathrm{~mm} \mathrm{~s}^{-1}$ for the second layer. To obtain welldefined pores, an extrusion flow of $75 \%$ and a gap of $0.65 \mathrm{~mm}$ were maintained between the rods. Using Simplify 3D software, 3D models were converted into optimized instructions to the printer to fabricate 3D printed PCL scaffolds.

Plasma coating of 3D printed scaffolds. 3D printed PCL scaffolds were cleaned in ethanol, dried with a nitrogen stream and placed in a custom-built plasma reactor chamber operated at $13.56 \mathrm{MHz} .{ }^{44}$ The scaffolds were then further cleaned using air plasma (50 W, 5 minutes, $1 \times 10^{-1}$ mbar) to remove unwanted organic materials. Plasma polymer coatings were applied in the presence of allylamine at $40 \mathrm{~W}$ for 2 minutes and at a precursor pressure of $1.3 \times 10^{-1} \mathrm{mbar}$. Before any further modification, the scaffolds were kept overnight at room temperature in vacuum-sealed containers to stabilize the plasma coating.

\section{Synthesis of AgNPs and subsequent immobilization}

AgNPs were synthesized using an established protocol by reducing silver nitrate with sodium borohydride. ${ }^{43} 12 \mathrm{ml}$ of $\mathrm{AgNO}_{3}$ ( $2 \mathrm{mM}$ ) was added to $5 \mathrm{ml}$ of mercaptosuccinic acid $(2 \mathrm{mM})$ under ice-cold conditions. Drops of $0.5 \mathrm{ml}$ of $\mathrm{NaBH}_{4}(0.5 \mathrm{M})$ were added slowly to the above mixture under vigorous stirring. The whole setup was protected from light and allowed to stir overnight until a stable reddish-brown solution of AgNPs was formed. The nanoparticle solution was stable for at least 6 months when stored in a refrigerator at $4{ }^{\circ} \mathrm{C}$.

Immobilization of AgNPs to the plasma polymer coated PCL scaffolds was carried out from solution for 6 hours (pPCL-Ag6) and 24 hours (pPCL-Ag24). After the immobilization period, the scaffolds were rinsed thrice in Milli-Q water and dried with a nitrogen stream. The scaffolds were placed in vacuum-sealed containers until further use.

\section{Scanning electron microscope (SEM)}

The morphologies of the control and test scaffolds were observed using a scanning electron microscope (Zeiss Merlin FEG-SEM, Zeiss, Jena, Germany) at $2 \mathrm{kV}$. The samples were mounted on aluminium stubs with double-sided tape and sputter-coated with platinum. From the images obtained, the average pore size was determined using ImageJ software.

\section{Ellipsometer}

The thickness of the plasma polymer was measured using a Variable Angle Spectroscopic Ellipsometer (A.J.A. Woollam Co. Inc.) fitted with a VB-400 control module and an HS-190 highspeed monochromator. WVASE32 software was used for data analysis. Three silicon wafers from each plasma deposition were used for thickness measurements and their average was recorded.

\section{Water contact angle analysis}

Sessile drop water contact angle measurements were carried out using a contact angle measurement instrument connected to a CCD camera (RD-SDMO2). The samples were placed on a plane solid support at room temperature. Measurements were taken by carefully adding $2 \mu \mathrm{L}$ of Milli-Q water on the surface of the scaffold at different positions and images of the droplet shape were captured every $30 \mathrm{~s}$. The contact angle was measured using the DropSnake plugin toolbar in ImageJ software. Triplicates of samples were analysed, and the mean of these values was tabulated.

\section{X-ray photoelectron spectroscopy (XPS)}

The chemical compositions of the modified and unmodified scaffolds were determined using a SPECS SAGE XPS equipped with a $\mathrm{Mg}$ radiation source that operates at $10 \mathrm{kV}$ and $20 \mathrm{~mA}$. For all the scaffolds, survey spectra were recorded over the binding energy range of $0-1000 \mathrm{eV}$ with $0.5 \mathrm{eV}$ resolution and a pass energy of $100 \mathrm{eV}$. The obtained spectra were processed and curve-fitted using CasaXPS software.

\section{Thermal analysis}

The thermal behaviour of the 3D printed samples was studied using a Differential Scanning Calorimeter (DSC, Q-20, TA Instruments). $5 \mathrm{mg}$ of each sample was weighed in aluminium pans and heated at $10{ }^{\circ} \mathrm{C} \min ^{-1}$ under a nitrogen flow at $50 \mathrm{~mL} \mathrm{~min}{ }^{-1}$ over the temperature range of 10 to $80{ }^{\circ} \mathrm{C}$. The melting and crystallization temperatures were estimated from the DSC graphs using the first heating scan. All the experiments were repeated thrice.

\section{Mechanical analysis}

The mechanical properties of the scaffolds were analyzed using a nano-indenter (Nano Guru, NG50, Instron) with a Berkovich diamond probe. The samples were fixed on the flat sample holder disc using glue and random test locations were chosen under an optical microscope by moving the translation stage. Displacement-controlled nanoindentation experiments were 
conducted using a trapezoidal loading curve with a loading rate of $30 \mathrm{~nm} \mathrm{~s}^{-1}$ for $5 \mathrm{~s}$ at a peak displacement of $150 \mathrm{~nm}$. All the experiments were repeated thrice.

\section{Antibacterial assay}

The anti-bacterial properties of AgNP loaded 3D printed scaffolds were investigated against Staphylococcus epidermidis (ATCC 35984) and Pseudomonas aeruginosa (NCTC 6749). These bacterial strains were chosen based on their ability to cause wound infections. Each scaffold was placed in a sterile well plate to which $1 \mathrm{ml}$ of Tryptone Soy Broth (TSB) was added and incubated at $37{ }^{\circ} \mathrm{C}$ on a shaker for 2 hours. To these wells, $10^{5} \mathrm{CFU} \mathrm{ml^{-1 }}$ of either $S$. epidermidis or $P$. aeruginosa were added and incubated overnight at $37{ }^{\circ} \mathrm{C}$ on a shaker. The control wells contained $1 \mathrm{ml}$ of TSB containing bacteria without any scaffolds. After incubation, TSB from each well was serially diluted and $10 \mu \mathrm{L}$ was plated onto agar plates and incubated overnight at $37{ }^{\circ} \mathrm{C}$. Duplicates of samples were analyzed and colony-forming units (CFU) were counted and plotted for each sample. All the experiments were repeated thrice.

\section{Cell viability}

The biocompatibility of the prepared scaffolds was evaluated using the resazurin assay, a fluorometric assay that measures the metabolic capacity of live cells. HFF cells were seeded at a density of $5 \times 10^{4}$ cells per well in 24-well plates and incubated overnight at $37^{\circ} \mathrm{C}$. The cell culture medium (DMEM) was then removed, and cells were washed with PBS. Fresh DMEM was added to the wells along with PCL, pPCL, pPCL-Ag6 and pPCLAg24 and cells were allowed to grow for another 24 hours after which DMEM was replaced with the medium containing $10 \%$ resazurin and incubated for 1 hour. Then, $100 \mu \mathrm{L}$ of the medium was transferred to 96-well plates and the fluorescence intensity was recorded using a microplate spectrophotometer set at an excitation wavelength of $544 \mathrm{~nm}$ and an emission wavelength of $590 \mathrm{~nm}$. Triplicates of samples were analysed, and data were plotted as mean \pm SD. The cell viability was calculated using the following equation:

Cell viability $(\%)=($ Fluorescence intensity of test scaffold $) /$ (Fluorescence intensity of control scaffold) $\times 100$

\section{Cell attachment}

For fixing HFF cells, $1.25 \%$ glutaraldehyde was used along with $4 \%$ paraformaldehyde and $4 \%$ sucrose in PBS. The fixative was removed and the cells were washed with PBS, dehydrated in $50 \%(\mathrm{v} / \mathrm{v})$ ethanol and then completely dried using hexamethyldisilazane (HMDS). The fixed cells were sputter-coated in platinum and imaged using a Zeiss Merlin FEG-SEM scanning electron microscope (Zeiss, Jena, Germany).

\section{Immune studies}

The monocyte cell lines (THP-1) were differentiated into macrophages using phorbol-12-myristate 13-acetate (PMA) and grown on 24-well plates at a density of $5 \times 10^{4}$ cells as reported previously. ${ }^{45,46}$ After overnight incubation, the cell culture medium (RPMI) was removed and the cells were washed with PBS. Fresh RPMI was added to the wells along with PCL, PPCL, pPCL-Ag6 and pPCL-Ag24. $1 \mu \mathrm{g} \mathrm{ml} \mathrm{m}^{-1}$ of LPS was added to the wells to activate macrophages and create an inflammatory environment and incubated for 6 hours after which the conditioned medium was collected and centrifuged to remove cell debris. The supernatant was then tested for IL-8, IL-1 $\beta$ and TNF $\alpha$ using ELISA kits (BioLegend, San Diego, CA, USA) following instructions from the manufacturer.

\section{In vivo studies}

Male Sprague Dawley rats (180-260 g) were selected from the inbred animals for the in vivo studies. The rats were obtained from Kerala Veterinary and Animal Sciences University, Mannuthy, India. All the animal procedures were performed with the approval of institutional animal ethics committee (No. 602/ $\mathrm{PO} / \mathrm{Re} / \mathrm{S} / 2002 / \mathrm{CPCSEA})$, strictly adhering to the guidelines of CPCSEA constituted by the Ministry of Environment, Forests and Climate Change, Animal Welfare Division of Government of India in Pushpagiri Institute of Medical Sciences and Research Centre, Tiruvalla, Kerala, India. The animal breeds were housed in sterile cages under aseptic conditions with provision of sterile food and water. The animals were anesthetized with a mixture of Ketamine Hydrochloride and Xylazine Injections, the dorsal region of the rats was shaved and sterilized with alcohol, two $1.5 \times 1.5 \mathrm{~cm}^{2}$ full thickness skin excision wounds were created and a skin flap was reflected to expose the subcutaneous tissue. The 3D scaffolds with $1.5 \times 1.5 \mathrm{~cm}^{2}$ dimensions were sutured on the wound. The membranes were implanted subcutaneously, and the skin flap was sutured back to retain the polymer membranes within the subcutaneous pouch. The skin pouch was reopened on day 7 and day 21 to evaluate the tissue response to the polymer membranes. Visual observation was done to find any signs of local inflammation caused by the surgical implantation. Hematoxylin and eosin stained sections were investigated for overall morphology, adhesion, and tissue response to the biomaterial. The stained sections (a minimum of 10 sections obtained from different levels of each tissue) were examined. Locations of inflammatory cells (macrophages, polymorphonuclear leukocytes, lymphocytes, and plasma cells), presence of giant cells, and the blood vessels were separately evaluated.

\section{Results and discussion}

To develop surface-engineered PCL scaffolds, a combination of $3 \mathrm{D}$ printing and plasma polymerisation was employed. In the current study, the $3 \mathrm{D}$ printed scaffolds $\left(18 \times 18 \times 0.4 \mathrm{~mm}^{3}\right)$ were fabricated using fused deposition modelling in which PCL was extruded as a thin filament through a heated nozzle and deposited via a 3D printer facilitated by Computer-Aided Design (Simplify3D) software. The scaffolds were prepared via layer-by-layer assembly in which the first layer of extruded PCL was fused with the second layer to form a porous structure for 


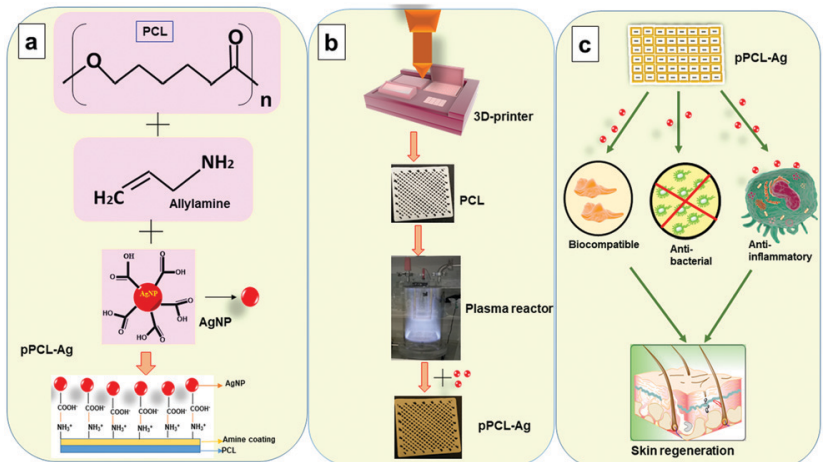

Fig. 1 Schematic representation of (a) the components involved in the fabrication of plasma polymer-modified PCL scaffolds containing AgNPs (pPCL-Ag) and their chemistry of immobilisation; (b) equipment and processes involved; and (c) targeted outcomes.

tissue regeneration. The resultant PCL scaffolds had an organised architecture achieved by a $45^{\circ}$ shift of the horizontal printing angle between successive layers. The obtained porous $3 \mathrm{D}$ constructs were then surface-modified by depositing a nanometer-thin layer from the plasma phase of vapour of allylamine (pPCL). The surface coatings had a thickness of $24.01 \pm 0.3 \mathrm{~nm}$ as evaluated by spectroscopic ellipsometry. Subsequently, AgNPs capped with MSA were immobilized to the plasma polymer layer. The AgNPs had an average particle diameter of $12 \pm 3 \mathrm{~nm}$, which is consistent with our previous reports (Fig. S1, ESI $\dagger$ ). ${ }^{45}$ The MSA molecule possessed two carboxylic acid groups which deprotonate in an aqueous medium. This allows electrostatic binding to the amine groups on the surface of the allylamine plasma polymer film which are protonated at neutral $\mathrm{pH}^{29}$ To control the AgNP surface concentration we immobilised the nanoparticles in a timedependent manner for 6 hours (pPCL-Ag6) and 24 hours (pPCL-Ag24), as schematically depicted in Fig. 1. After immobilization, the scaffolds adopted a light brownish colour due to the localised surface plasmon resonance of the AgNPs. ${ }^{47}$

\section{Surface morphology analysis}

The surface morphology of the fabricated scaffolds was evaluated using SEM. Fig. 2 shows hierarchical structures composed of well-ordered and interconnected pores. Pore

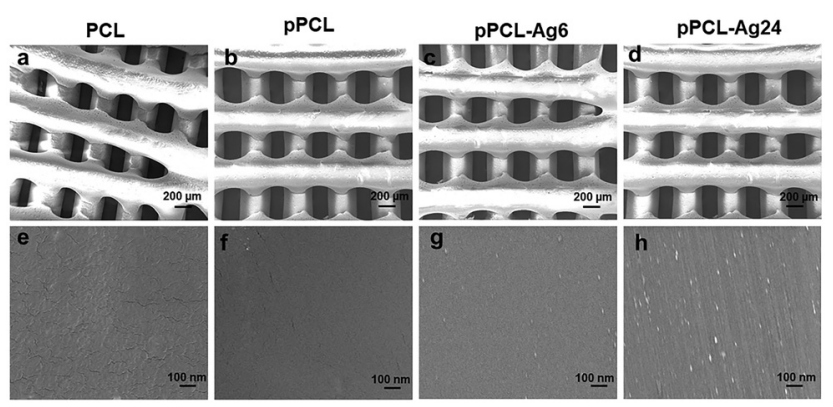

Fig. 2 SEM images of PCL, PPCL-Ag6, and PPCL-Ag24 at (a-d) lower and $(e-h)$ higher magnifications. interconnectivity has a positive impact on the rate of cell growth, depth of cell infiltration and migration. ${ }^{48}$ These pores are intended to facilitate efficient diffusion of nutrients and removal of waste products from cells. ${ }^{4,49}$ The average pore diameters of PCL, pPCL, pPCL-Ag6 and pPCL-Ag24 were $336 \pm 20 \mu \mathrm{m}, 345 \pm 36 \mu \mathrm{m}, 329 \pm 17 \mu \mathrm{m}$ and $327 \pm 20 \mu \mathrm{m}$, respectively. The SEM images indicate that neither the plasma polymerisation process nor the subsequent immobilization of AgNPs did not cause any critical changes to the bulk morphological properties or pore size of the original PCL scaffold (Fig. 2a-d). The pore sizes remained in the range required for promotion of cell proliferation, angiogenesis and penetration of tissues into the scaffolds. ${ }^{50}$ The high magnification SEM images (Fig. 2e and f) also revealed the smooth surface of the pristine PCL and PPCL scaffolds. Silver nanoparticles could also be visualized (Fig. $2 \mathrm{~g}$ and $\mathrm{h}$ ) pointing to an increase in the surface density of nanoparticles with increasing immobilization time. The average numbers of nanoparticles on PPCL-Ag6 and PPCL-Ag24 were $27 \pm 2$ and $82 \pm 1.5$ per $\mu \mathrm{m}^{2}$, respectively.

\section{Surface chemistry and wettability}

Biomaterial surface chemistry plays a critical role in governing subsequent biological responses. The surface chemical compositions of the modified and pristine PCL were evaluated by X-ray photoelectron spectroscopy (XPS). The survey spectra of the pristine PCL scaffold showed two peaks corresponding to C $1 \mathrm{~s}$ at $285 \mathrm{eV}$ and $\mathrm{O} 1 \mathrm{~s}$ at $532 \mathrm{eV}$ (Fig. 3A), consistent with the chemical structure of the polymer. When the scaffolds were coated with an allylamine based plasma polymer, a distinct peak corresponding to $\mathrm{N} 1 \mathrm{~s}$ at about $400 \mathrm{eV}$ appeared in the survey spectrum confirming the successful surface modification of the 3D printed PCL structures. After immobilization of AgNPs, an intense peak at $368 \mathrm{eV}$ corresponding to $\mathrm{Ag} 3 \mathrm{~d}$ was
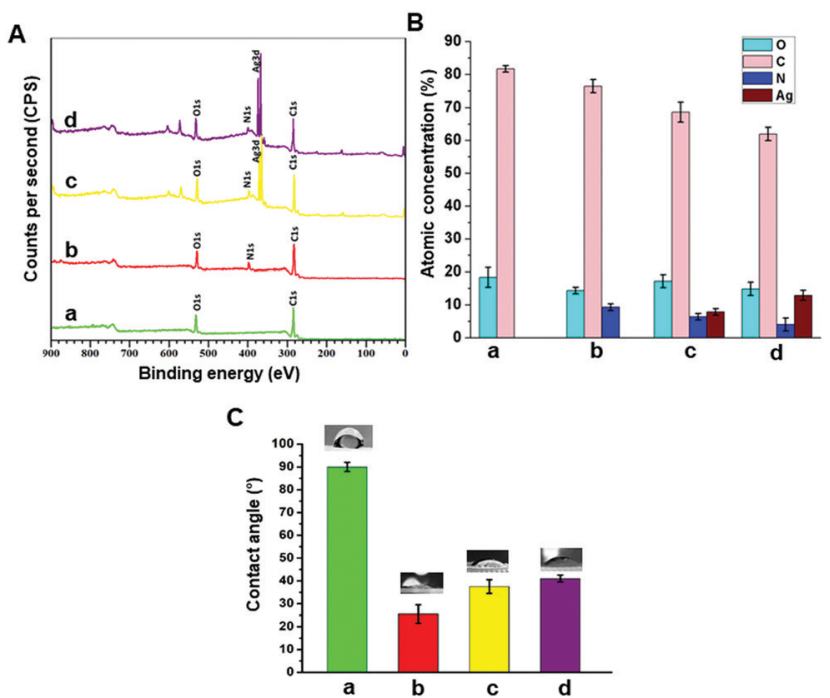

Fig. 3 Evaluation of the surface chemistry and surface wettability of (a) $\mathrm{PCL}$, (b) $\mathrm{pPCL}$, (c) $\mathrm{pPCL}-\mathrm{Ag} 6$, and (d) $\mathrm{pPCL}-\mathrm{Ag} 24$ from (A) X-ray photoelectron spectroscopy survey spectra, (B) quantification of the atomic percentage of elements identified in the survey spectra and (C) sessile drop water contact angle analysis. 
detectable (Fig. 3A). The atomic percent of silver on the surface of pPCL-Ag6, pPCL-Ag24 was $7.87 \pm 1 \%$ and $13.9 \pm 1.5 \%$, respectively (Fig. 3B).

Surface wettability is an influential factor that controls cell adhesion. ${ }^{51}$ Anchorage-dependent mammalian cells strongly favour hydrophilic surfaces for attachment and proliferation. $^{52}$ Wettability is dependent on surface topographical features and surface chemistry. ${ }^{53}$ Here, the static water contact angle was calculated as an indicator of the scaffold's wettability. The pristine PCL printed scaffold is hydrophobic with a water contact angle of WCA $=90 \pm 0.2^{\circ}$ (Fig. 3C). The hydrophobicity of PCL is due to the presence of hydrocarbon chains as PCL is mainly a saturated aliphatic polyester with many repeating units of hexanoate. ${ }^{54}$ As shown in Fig. 3C, after surface modification, the hydrophilicity of the surface significantly increased leading to a lower WCA in the range of $30-40^{\circ}$. The calculated WCA is a complex function of change in surface chemistry due to the introduction of polar amine groups on the surface by plasma coating and variation of surface topography by nanoparticle immobilization.

\section{Thermal and mechanical properties}

The thermal behaviour of the modified and unmodified PCL scaffolds was investigated using DSC analysis (Fig. 4A and B). The exothermic peak during cooling (Fig. 4B) corresponds to crystallization, while the endothermic peak (Fig. 4A) is due to melting. The melting $\left(T_{\mathrm{m}}\right)$ and crystallization $\left(T_{\mathrm{c}}\right)$ temperatures obtained from the DSC thermograms are listed in Table S1 (ESI $\dagger$ ). PCL is a semi-crystalline polymer having both amorphous and crystalline regions. The characteristic $T_{\mathrm{c}}$ and $T_{\mathrm{m}}$ of pristine PCL were 40.26 and $62.94{ }^{\circ} \mathrm{C}$, respectively. No significant difference in the $T_{\mathrm{c}}$ was observed for the scaffolds before
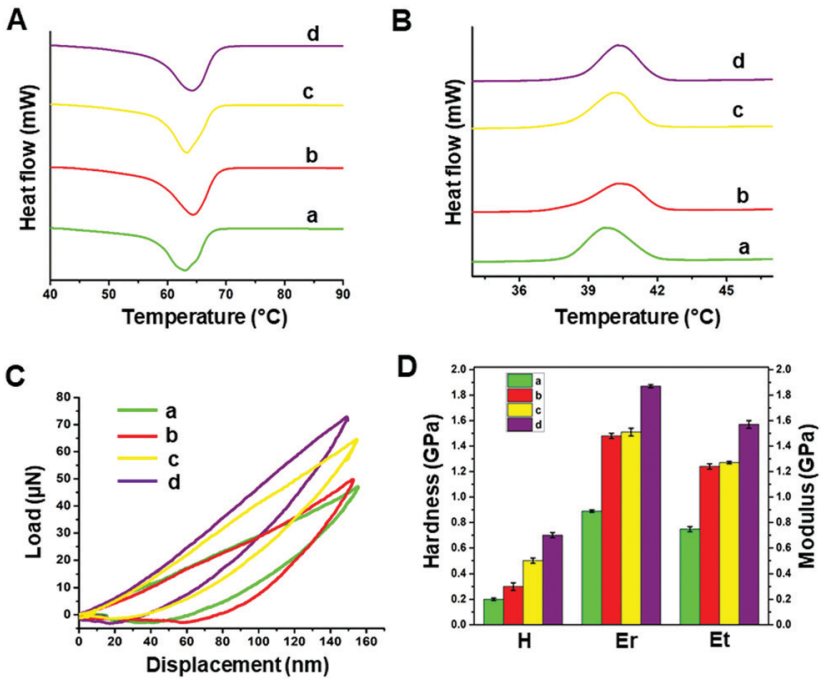

Fig. 4 Assessment of the thermal and mechanical properties of (a) PCL, (b) $\mathrm{pPCL}$, (c) pPCL-Ag6 and (d) pPCL-Ag24 from the (A) DSC melting thermogram, (B) DSC crystallization thermogram, (C) nanoindentation load-displacement curves at a peak displacement of $150 \mathrm{~nm}$ and (D) comparison of hardness $(H)$, reduced modulus $(E r)$ and modulus of elasticity (Et). and after surface modification. However, there was a slight increase in the $T_{\mathrm{m}}$ of the plasma coated scaffolds (PPCL, pPCL-6, pPCL-24) compared to pristine PCL. Tm is the temperature at which the molecular chains of PCL lose their regular well-defined orientation and become disordered. The small increase in Tm could be attributed to the surface modification which restricted the motion of molecular chains at the surface of the material, requiring more heat to melt the crystalline regions in scaffolds. ${ }^{55,56}$

The mechanical properties of PCL, pPCL, pPCL-Ag6 and pPCL-Ag24 were evaluated using nano-indentation at a displacement of $150 \mathrm{~nm}$ (Fig. 4C and D). During the nano-indentation test, the nano-indenter tip penetrates through the surface of the test material and records force and displacement during the loading and unloading regime. The resulting load-displacement curve is the response of interest from which nanomechanical properties such as hardness and modulus are elucidated. The Oliver-Pharr method was used to calculate the displacement controlled reduced modulus and hardness of the samples. The reduced modulus was calculated from eqn (1):

$$
\operatorname{Er}=S(\sqrt{\pi} /(2 \sqrt{\mathrm{Ap}}))
$$

where $S$ is the unloading stiffness and Ap is the projected area.

Modulus of elasticity (Et) can be deduced from Er using eqn (2):

$$
1 / \operatorname{Er}=\left(1-\nu_{1}\right) / E_{1}+\left(1-\nu_{\mathrm{t}}\right) / E_{\mathrm{t}}
$$

In eqn (2), $E_{1}$ and $\nu_{1}$ are the modulus and Poisson ratio of the diamond indenter, respectively. $E_{\mathrm{t}}$ and $\nu_{\mathrm{t}}$ are the modulus and Poisson ratio of the test material, respectively. For a diamond indenter probe, the values of $\nu_{1}$ and $E_{1}$ are 0.07 and $1140 \mathrm{GPa}$, respectively. $\nu_{\mathrm{t}}$ is presumed to be 0.4 from a rough estimation. ${ }^{57}$ Using eqn (2), the $E_{\mathrm{t}}$ values for PCL, PPCL, pPCL-Ag6 and pPCL-Ag24 were calculated and are plotted in Fig. 4D.

As it can be seen from the load-displacement curves, there was a substantial increase in the required load to obtain the same displacement in the case of the surface modified scaffolds compared to the pristine material (Fig. 4C). Both the hardness and modulus of the scaffolds increased for plasma polymer-modified scaffolds (Fig. 4D). This was because, when indented, both the plasma polymer and the underlying substrate contribute to the deformation process. After immobilization of AgNPs onto the plasma coated scaffolds, the indenter experienced more resistance as it penetrated the surface to a depth of $150 \mathrm{~nm}$. The nano-mechanical evaluation demonstrates that pPCL-6 $\mathrm{h}$ and pPCL-24 $\mathrm{h}$ were mechanically robust. The reduced modulus of unmodified PCL was $0.889 \pm$ $0.017 \mathrm{GPa}$, and that of plasma modified PCL was $1.48 \pm 0.021$ GPa. 3D scaffolds immobilized with silver nanoparticles had a reduced modulus of $1.51 \pm 0.03 \mathrm{GPa}$. With an increase in immobilisation time of silver nanoparticles to 24 hours, there was an increase in reduced modulus to $1.87 \pm 0.012 \mathrm{GPa}$. This may be because more nanoparticles were attached onto the 
surface after 24 hours as quantified by XPS, which offered more resistance to the indenter.

\section{Antibacterial properties}

The antibacterial efficacy of the prepared scaffolds was evaluated against $S$. epidermidis (SE) ATCC 35984 (Gram-positive) and $P$. aeruginosa (PA) NCTC 6749 (Gram-negative) as these bacteria are often associated with wound infections and are representative of Gram-positive and Gram-negative species, respectively. ${ }^{58,59}$ Antibacterial testing was carried out by first incubating the scaffolds in $10^{5} \mathrm{CFU} \mathrm{ml}^{-1}$ of bacteria for $24 \mathrm{~h}$ at $37{ }^{\circ} \mathrm{C}$. The resulting bacterial suspensions were plated on agar plates, and the colonies were counted to evaluate the number of viable bacteria. The average colonies counted on SE and

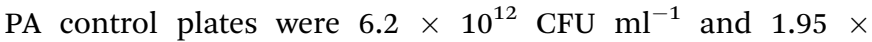
$10^{13} \mathrm{CFU} \mathrm{m}{ }^{-1}$, respectively (Fig. 5). However, no bacterial colony growth was observed when both bacterial species were incubated with pPCL-Ag6 and pPCL-Ag24 modified scaffolds. This indicates that modification of the PCL scaffolds with silver nanoparticles can provide effective protection against infection. Our results were consistent with a body of knowledge on the antibacterial properties of silver nanoparticles, a topic that generated tremendous interest over the last two decades. Upon exposure to an aqueous medium, AgNPs oxidise which leads to the release of silver ions that bind to bacterial DNA, block replication and cell metabolism and cause bacterial lysis. ${ }^{35,60}$

\section{Biocompatibility and immune responses}

The biocompatibility of the nanocomposite scaffolds was tested with primary human foreskin fibroblasts via the resazurin assay. Fibroblasts were selected for evaluating biocompatibility as these cells play a principal role in wound healing and tissue regeneration. ${ }^{61}$ In this assay, the metabolic activity of the cells is quantified in terms of the fluorescence intensity of resorufin. Resazurin is converted to irreversible fluorescent pink coloured resorufin by viable cells. The results are presented in Fig. 6B and showed that functionalised PCL scaffolds exhibited
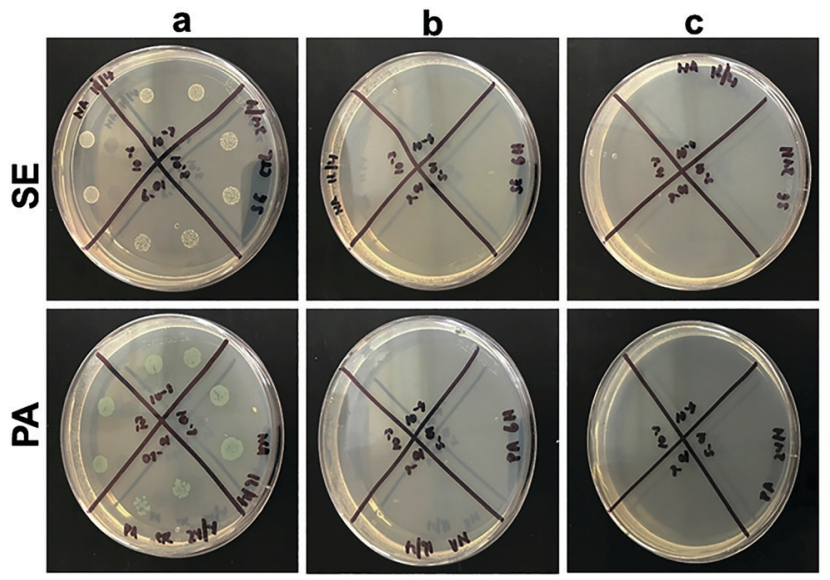

Fig. 5 Photographs of bacterial colonies (Staphylococcus epidermidis, $\mathrm{SE}$, and Pseudomonas aeruginosa, PA) grown on agar Petri dishes for (a) control and after incubation with (b) pPCL-Ag6 and (c) pPCL-Ag24.

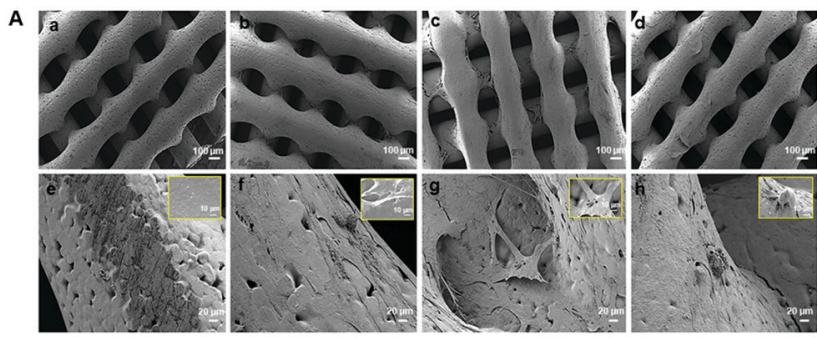

B

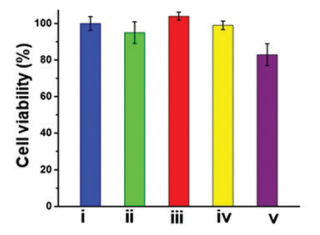

Fig. 6 (A) Cell attachment on (a and e) PCL, (b and f) pPCL, (c and g) pPCL-Ag6 and (d and h) pPCL-Ag24 at lower (a-d) and higher (e-h) magnifications. The inset figures show the higher magnification images of the attached single cell on respective scaffolds to show its metabolic state. (B) The biocompatibility of (i) control, (ii) PCL, (iii) pPCL, (iv) pPCL-Ag6 and (v) $\mathrm{PPCL}-\mathrm{Ag} 24$ (statistical significance was estimated using one-way ANOVA; $p<0.0001)$

negligible cytotoxicity. A small reduction in metabolic activity was observed for pPCL-Ag24 scaffolds. However, even in the case of the highest silver nanoparticle surface concentration (pPCL-Ag24) the cell metabolic activity was well within $80 \%$ of control which is accepted as satisfactory by ISO 109993-5 standards. ${ }^{62}$

The morphology of the cells attached on the scaffold surface was assessed using scanning electron microscopy after 48 hours of cell culture (Fig. 6A). The results conveyed that the distribution and spreading of cells are more homogenous in the composite scaffolds compared to native PCL scaffolds. Hydrophobicity and low surface energy could be the possible reasons that are attributed to the poor cell infiltration on native PCL scaffolds. ${ }^{13-16}$ Cell bridging was evident in PPCL, PPCLAg6 and pPCL-Ag24 scaffolds exhibiting strong cell-cell communications. Most of the cells were found to strongly anchor to the fist layer of functionalised scaffolds and gradually migrate to the top layer in 48 hours. In PPCL and pPCL-Ag6, cells showed a spread morphology, indicating the active physiological state of the cells (inset figures). This was supported by the presence of protrusions called lamellipodia seen in active cells showing the well-established attachment of cells. Cell proliferation was comparatively less on pPCL-Ag24 scaffolds, in agreement with the viability results discussed above. The results of the test suggested that modification of PCL scaffolds with AgNPs is a viable pathway to reduce the risk of infection while retaining the biocompatibility of the material.

In the next step, we evaluated the innate immune response of the modified and unmodified PCL scaffolds. The immune system plays a pivotal role in wound healing and repair, and thus it is important to assess whether the modified scaffolds may elicit an undesired immunological response. ${ }^{63,64}$ Impaired healing of wounds is often associated with raised levels of pro-inflammatory cytokines and prolonged inflammatory phase. ${ }^{65,66}$ A good dermal 
A

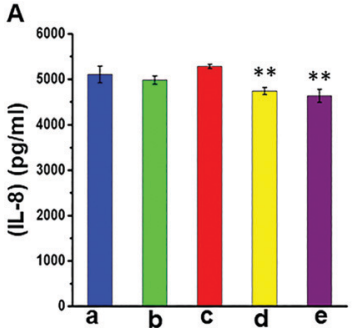

B

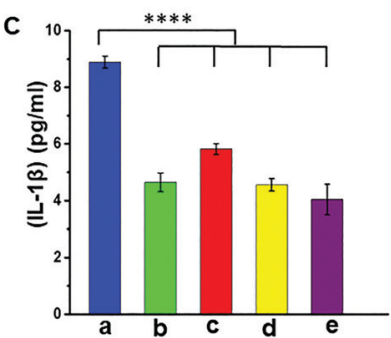

Fig. 7 Evaluation of the immune response. Pro-inflammatory cytokine expression: (A) IL-8, (B) TNF- $\alpha$ and (C) IL-1 $\beta$ from differentiated THP-1 cells for (a) control, (b) $\mathrm{PCL}$, (c) $\mathrm{pPCL}$, (d) $\mathrm{pPCL}-\mathrm{Ag} 6$ and (e) $\mathrm{pPCL}-\mathrm{Ag} 24$ (statistical significance was estimated using one-way ANOVA; ${ }^{* * * *} p<0.0001,{ }^{* * *} p<0.001,{ }^{* *} p<0.01$ ).

tissue engineering scaffold is expected to reduce inflammation and promote skin regeneration.

In this study, we differentiated monocyte like cell lines (THP-1) into macrophages which were used as model cells to investigate the immune responses. The modified and unmodified scaffolds were incubated in the culture of these cells overnight at $37{ }^{\circ} \mathrm{C}$ to determine the level of expression of proinflammatory markers as a function of surface modification and the presence of AgNPs. The results from ELISA analysis (Fig. 7) showed that there was little difference in the expression of IL-8 (a neutrophil recruitment mediator) in all groups, with only a small (but a statistically significant) reduction in the case of pPCL-Ag6 and pPCL-Ag24. The expression of IL-1 $\beta$ was very similar for the modified and unmodified scaffolds, with the small differences in the cases of pPCL-Ag6 and pPCL-Ag24 being not statistically significant. However, there was a major reduction in the expression of TNF- $\alpha$ in the silver nanoparticle coated groups. TNF- $\alpha$ is a proinflammatory cytokine and a major driver of many signalling events leading to the recruitment of inflammatory cells and amplification of the inflammatory response. While inflammation is an important part of the defence response to invading pathogens, many studies demonstrate that the process delays wound healing. For example, Ritsu et al. demonstrated that suppression of TNF- $\alpha$ expression led to faster wound healing. ${ }^{67}$ Thus, we speculate that the reduction observed in the expression of pro-inflammatory cytokines in the case of scaffolds containing silver nanoparticles, and in particular TNF- $\alpha$, may facilitate faster-wound healing.

\section{In vivo implantation studies}

Normally a foreign body implanted in vivo can elicit a strong inflammatory response. A moderate pro-inflammatory response encourages wound healing, but an excessive inflammatory response leads to organ damage and dysfunction. Hence it is important to analyze the in vivo performance of the scaffolds after implantation (Fig. 8C). No evident adverse inflammatory reaction (infection, fistula, or fibrous capsule) was observed at either the implantation sites or adjacent sites during the post-transplantation follow-up period of 21 days (Fig. 8A). The formation of new blood vessels (angiogenesis) is visible in all samples (Fig. 8B).

From the histological images, it is evident that the PCL scaffolds containing AgNPs enhanced the proliferation of fibroblasts compared to PCL and PPCL (Fig. 9). During wound healing, the fibroblasts proliferate and secrete various extracellular matrix (ECM) proteins including fibrin, fibronectin, collagen, and other ECM proteins that provide a provisional matrix for angiogenesis and tissue remodelling. This leads to the development of granulation tissue, which is imperative for proper wound healing. You et al. reported that AgNPs accelerated the migration of fibroblasts with an increased expression of $\alpha$-smooth muscle actin ( $\alpha$-SMA). ${ }^{68}$ Neat PCL was characterized by a large number of giant cells and plasma cells (chronic inflammatory cells) which indicates inflammation. Numerous plasma cells (chronic inflammatory cells) were observed in neat PCL after seven days which shows inflammation. On the 21st day, fibroblast proliferation and angiogenesis were seen in the PCL group along with foreign body reaction. In the plasma modified group, edema was more pronounced on the 7 th-day. This indicates that vascular proliferation is yet to start and is predominated by early vessels having leaky nature. Overall response is delayed in the amine-modified group. Even on the 21st day, the edema continued along with plasma cells and no evident fibroblast proliferation could be seen. A large number of fibroblasts could proliferate through pPCL-Ag6 with

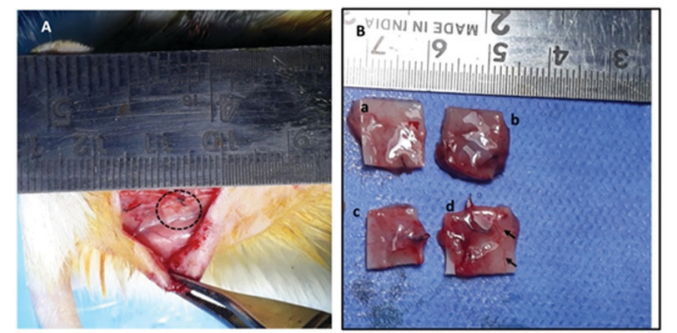

c

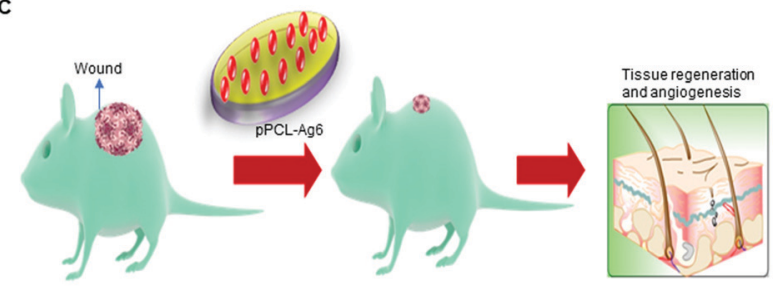

Fig. 8 (A) 3D printed samples were integrated into the host tissue (dotted circle). (B) Tissues integrated with (a) PCL, (b) pPCL, (c) pPCL-Ag6 and (d) pPCL-Ag24; angiogenesis (formation of new blood vessels) is evidently seen in PCL-Ag samples (d). (C) Schematic representation showing the in vivo performance of scaffolds. 


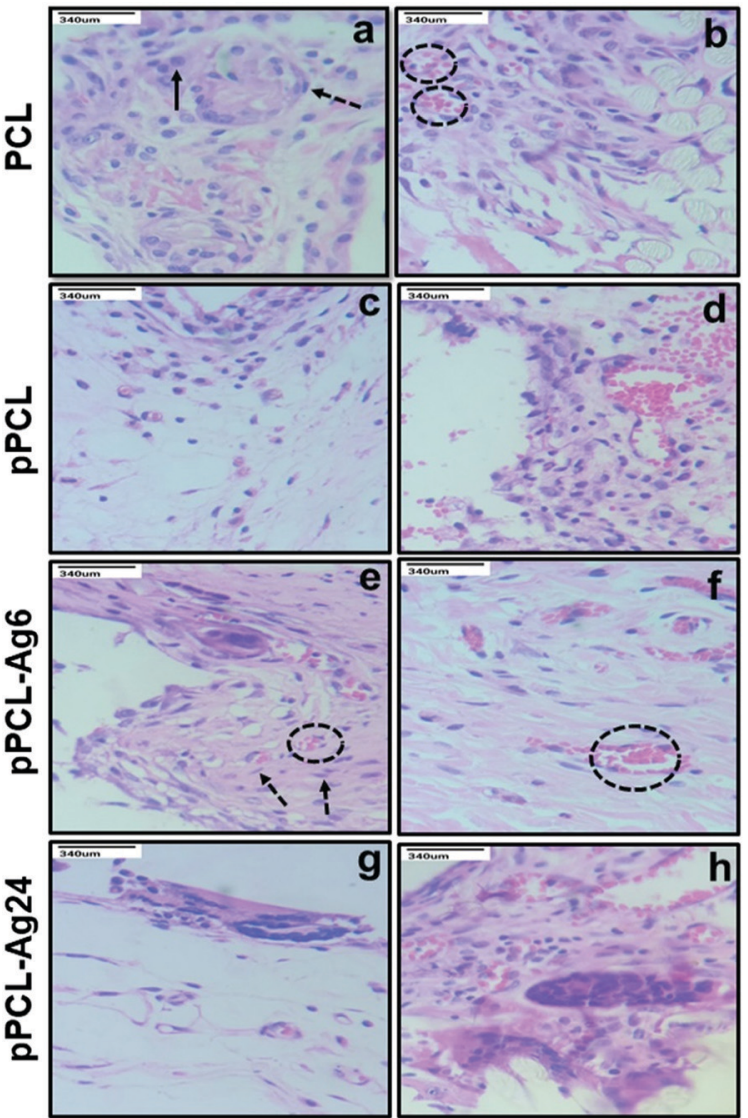

Fig. 9 Histological analysis of (a) PCL, (c) pPCL, (e) pPCL-Ag6 and (g) pPCL-Ag24 after seven days of implantation and (b) PCL, (d) PPCL, (f) pPCL-Ag6 and (h) pPCL-Ag24 after 21 days of implantation (dotted arrows indicate fibroblasts, bold arrow shows neutrophils and dotted circles refer to angiogenesis).

a few giant cells which shows that incorporation of silver nanoparticles did not induce much inflammation when compared to PCL and PPCL. On the 7th day, collagen formation was seen with vessel proliferation and no edema was observed. In the pPCL-Ag6 group, collagen deposition was seen and the vessels were also matured on the 21st day. Fibroblast cells were also observed with minimum inflammation. In the pPCL-Ag24 group, foreign body reaction was observed along with minimum angiogenesis and collagen deposition was also low on the 7th day. Fibroblast proliferation and collagen deposition are seen on the 21st day. However inflammatory reaction is still predominant which delays wound healing. This could be due to the increased concentration of silver nanoparticles in 3D printed PCL incubated in silver nanoparticles for 24 hours. Samberg et al. reported increased edema and focal inflammation with increased silver nanoparticle concentration. ${ }^{69}$ Hence, we propose the PPCL-Ag6 scaffold as a suitable material for tissue engineering applications.

\section{Conclusions}

In the present study, we demonstrate a plasma nanoengineering approach to fabricate 3D PCL printed scaffolds with improved mechanical and antibacterial properties. The surface properties of the PCL scaffolds were successfully tailored via a thin plasma polymer film deposited from the vapour of allylamine. The positive charge of the protonated amine groups available in the plasma polymer coating was utilised for the electrostatic immobilization of silver nanoparticles in a concentration-controlled manner. The scaffolds demonstrated an improvement in mechanical properties after AgNP immobilization. The AgNP functionalised scaffolds showed complete inhibition of the growth of Gram-positive and Gram-negative pathogens often associated with wound infections. Studies with primary human dermal fibroblasts showed no apparent cytotoxicity caused by the plasma polymer coating or the incorporated AgNPs. We also examined the immune responses in a culture of macrophages obtained from differentiated THP-1 human monocyte cell lines. We found a reduction of proinflammatory cytokine expression by the scaffolds containing AgNPs. The most significant reduction was in the secretion of TNF- $\alpha$, a cytokine associated with amplification of inflammation and delayed wound healing. The scaffolds that were incubated in AgNPs for 6 hours were more biocompatible than scaffolds incubated for 24 hours. Mature blood vessels were also seen in 6 hour incubated scaffolds in our in vivo experiments. Thus, the surface plasma polymer facilitated nanoengineering strategy for modification of 3D tissue engineering scaffolds presented in this work demonstrates the possibility not only for protecting from infection but also for accelerating wound healing. The outcomes of this study provide new surface engineering opportunities that can be adopted in the field for refining the properties of biomaterial constructs.

\section{Author contributions}

Neethu Ninan: conceptualization, methodology, software, formal analysis, investigation, supervision, writing - original draft. Blessy Joseph: methodology, software, investigation, formal analysis. Rahul Madathiparambil Visalakshan: methodology, software, investigation, formal analysis. Richard Bright: methodology, formal analysis, investigation. Clement Denoual: methodology, software, investigation. Nandakumar Kalarikkal: writing - review \& editing. Peter Zilm: writing - review \& editing, resources. Yves Grohens: resources, writing - review \& editing. Sabu Thomas: conceptualization, writing review \& editing. Krasimir Vasilev: conceptualization, writing review \& editing, supervision, resources, funding acquisition, validation. Yogesh Bharat Dalwi: formal analysis, investigation. Priya P. V.: methodology, formal analysis. Aji Mathew: conceptualization, writing - review \& editing.

\section{Conflicts of interest}

There are no conflicts to declare.

\section{Acknowledgements}

Krasimir Vasilev thanks ARC for DP15104212, NHMRC for fellowship APP1122825, and Project grant APP1032738 and 
Alexander von Humboldt Foundation for Fellowship for Experienced Researchers. This work was also supported by Mahatma Gandhi University Junior Research fellowship (529/A6/2/JRF 2018-19/Ac).

\section{References}

1 G. M. Cunniffe, P. J. Díaz-Payno, E. J. Sheehy, S. E. Critchley, H. V. Almeida, P. Pitacco, S. F. Carroll, O. R. Mahon, A. Dunne, T. J. Levingstone, C. J. Moran, R. T. Brady, F. J. O'Brien, P. A. J. Brama and D. J. Kelly, Biomaterials, 2019, 188, 63-73.

2 A. Li, Y. Wei, C. Hung and G. Vunjak-Novakovic, Biomaterials, 2018, 173, 47-57.

3 M. Xue and C. J. Jackson, Adv. Wound Care, 2015, 4, 119-136.

4 N. Ninan, Y. Grohens, A. Elain, N. Kalarikkal and S. Thomas, Eur. Polym. J., 2013, 49, 2433-2445.

5 N. Ninan, M. Muthiah, N. A. Bt. Yahaya, I.-K. Park, A. Elain, T. W. Wong, S. Thomas and Y. Grohens, Colloids Surf., B, 2014, 115, 244-252.

6 N. Ninan, M. Muthiah, I.-K. Park, A. Elain, S. Thomas and Y. Grohens, Carbohydr. Polym., 2013, 98, 877-885.

7 L. Moroni, J. A. Burdick, C. Highley, S. J. Lee, Y. Morimoto, S. Takeuchi and J. J. Yoo, Nat. Rev. Mater., 2018, 3, 21-37.

8 A. A. Giannopoulos, D. Mitsouras, S.-J. Yoo, P. P. Liu, Y. S. Chatzizisis and F. J. Rybicki, Nat. Rev. Cardiol., 2016, 13, 701.

9 D. Liu, W. Nie, D. Li, W. Wang, L. Zheng, J. Zhang, J. Zhang, C. Peng, X. Mo and C. He, Chem. Eng. J., 2019, 362, 269-279.

10 S. Yuan, G. Xiong, F. He, W. Jiang, B. Liang and C. Choong, J. Mater. Chem. B, 2015, 3, 8088-8101.

11 J. C. Middleton and A. J. Tipton, Biomaterials, 2000, 21, 2335-2346.

12 O. S. Manoukian, M. R. Arul, N. Sardashti, T. Stedman, R. James, S. Rudraiah and S. G. Kumbar, J. Appl. Polym. Sci., 2018, 135, 46068.

13 Y. Zhu, C. Gao and J. Shen, Biomaterials, 2002, 23, 4889-4895.

14 M. Diba, M. Kharaziha, M. H. Fathi, M. Gholipourmalekabadi and A. Samadikuchaksaraei, Compos. Sci. Technol., 2012, 72, 716-723.

15 Y. Zhu, C. Gao, X. Liu and J. Shen, Biomacromolecules, 2002, 3, 1312-1319.

16 S. Miroshnichenko, V. Timofeeva, E. Permykova, S. Ershov, P. Kiryukhantsev-Korneev, E. Dvořaková, D. V. Shtansky, L. Zajíčková, A. Solovieva and A. Manakhov, Nanomaterials, 2019, 9, 637.

17 A. Manakhov, E. Kedroňová, J. Medalová, P. Černochová, A. Obrusník, M. Michlíček, D. V. Shtansky and L. Zajíčková, Mater. Des., 2017, 132, 257-265.

18 A. A. D'souza and R. Shegokar, Expert Opin. Drug Delivery, 2016, 13, 1257-1275.

19 B. Akhavan, M. Croes, S. G. Wise, C. Zhai, J. Hung, C. Stewart, M. Ionescu, H. Weinans, Y. Gan, S. Amin Yavari and M. M. M. Bilek, Appl. Mater. Today, 2019, 16, 456-473.
20 F. Yang, J. G. C. Wolke and J. A. Jansen, Chem. Eng. J., 2008, 137, 154-161.

21 S. E. Kim, C.-S. Kim, Y.-P. Yun, D. H. Yang, K. Park, S. E. Kim, C.-M. Jeong and J.-B. Huh, Carbohydr. Polym., 2014, 114, 123-132.

22 A. A. Cavallaro, M. N. Macgregor-Ramiasa and K. Vasilev, ACS Appl. Mater. Interfaces, 2016, 8, 6354-6362.

23 K. Vasilev, V. Sah, K. Anselme, C. Ndi, M. Mateescu, B. Dollmann, P. Martinek, H. Ys, L. Ploux and H. J. Griesser, Nano Lett., 2010, 10, 202-207.

24 Z. Chen, A. Bachhuka, S. Han, F. Wei, S. Lu, R. M. Visalakshan, K. Vasilev and Y. Xiao, ACS Nano, 2017, 11, 4494-4506.

25 M. MacGregor-Ramiasa, I. Hopp, A. Bachhuka, P. Murray and K. Vasilev, Acta Biomater., 2017, 56, 171-180.

26 J. L. Hernandez-Lopez, R. E. Bauer, W. S. Chang, G. Glasser, D. Grebel-Koehler, M. Klapper, M. Kreiter, J. Leclaire, J. P. Majoral, S. Mittler, K. Müllen, K. Vasilev, T. Weil, J. Wu, T. Zhu and W. Knoll, Mater. Sci. Eng., C, 2003, 23, 267-274.

27 M. N. Macgregor-Ramiasa, A. A. Cavallaro and K. Vasilev, J. Mater. Chem. B, 2015, 3, 6327-6337.

28 R. V. Goreham, A. Mierczynska, M. Pierce, R. D. Short, S. Taheri, A. Bachhuka, A. Cavallaro, L. E. Smith and K. Vasilev, Thin Solid Films, 2013, 528, 106-110.

29 S. N. Christo, A. Bachhuka, K. R. Diener, A. Mierczynska, J. D. Hayball and K. Vasilev, Adv. Healthcare Mater., 2016, 5, 956-965.

30 P. Cools, H. Declercq, R. Ghobeira, R. Morent and N. De Geyter, Surf. Coat. Technol., 2018, 350, 925-935.

31 K. Vasilev, J. Cook and H. J. Griesser, Expert Rev. Med. Devices, 2009, 6, 553-567.

32 K. Vasilev, Coatings, 2019, 9, 654.

33 W. Zheng, Y. Jia, W. Chen, G. Wang, X. Guo and X. Jiang, ACS Appl. Mater. Interfaces, 2017, 9, 21181-21189.

34 C. R. Arciola, D. Campoccia and L. Montanaro, Nat. Rev. Microbiol., 2018, 16, 397-409.

35 L. E. González García, M. N. MacGregor, R. M. Visalakshan, N. Ninan, A. A. Cavallaro, A. D. Trinidad, Y. Zhao, A. J. D. Hayball and K. Vasilev, Chem. Commun., 2019, 55, 171-174.

36 S. Taheri, G. Baier, P. Majewski, M. Barton, R. Förch, K. Landfester and K. Vasilev, J. Mater. Chem. B, 2014, 2, 1838-1845.

37 Z. Muwaffak, A. Goyanes, V. Clark, A. W. Basit, S. T. Hilton and S. Gaisford, Int. J. Pharm., 2017, 527, 161-170.

38 K. Kalwar, W.-X. Sun, D.-L. Li, X.-J. Zhang and D. Shan, React. Funct. Polym., 2016, 107, 87-92.

39 C. Liao, Y. Li and S. C. Tjong, Nanomaterials, 2019, 9(7), 952.

40 R. Thomas, K. R. Soumya, J. Mathew and E. K. Radhakrishnan, Appl. Biochem. Biotechnol., 2015, 176, 2213-2224.

41 S. Radhakrishnan, S. Nagarajan, H. Belaid, C. Farha, I. Iatsunskyi, E. Coy, L. Soussan, V. Huon, J. Bares, K. Belkacemi, C. Teyssier, S. Balme, P. Miele, D. Cornu, N. Kalkura, V. Cavaillès and M. Bechelany, Mater. Sci. Eng., C, 2021, 118, 111525 . 
42 Y. Gao, A. M. Hassanbhai, J. Lim, L. Wang and C. Xu, $R S C$ Adv., 2017, 7, 10051-10056.

43 C. M. B. Ho, A. Mishra, P. T. P. Lin, S. H. Ng, W. Y. Yeong, Y.-J. Kim and Y.-J. Yoon, Macromol. Biosci., 2017, 17, 1600250.

44 R. M. Visalakshan, A. A. Cavallaro, M. N. MacGregor, E. P. Lawrence, K. Koynov, J. D. Hayball and K. Vasilev, Adv. Funct. Mater., 2019, 29, 1807453.

45 S. Taheri, A. Cavallaro, S. N. Christo, L. E. Smith, P. Majewski, M. Barton, J. D. Hayball and K. Vasilev, Biomaterials, 2014, 35, 4601-4609.

46 W. Chanput, J. J. Mes, H. F. J. Savelkoul and H. J. Wichers, Food Funct., 2013, 4, 266-276.

47 K. Shrivas, S. Sahu, G. K. Patra, N. K. Jaiswal and R. Shankar, Anal. Methods, 2016, 8, 2088-2096.

48 N. Ninan, A. Forget, V. P. Shastri, N. H. Voelcker and A. Blencowe, ACS Appl. Mater. Interfaces, 2016, 8, 28511-28521.

49 N. Ninan, M. Muthiah, I.-K. Park, A. Elain, T. W. Wong, S. Thomas and Y. Grohens, ACS Appl. Mater. Interfaces, 2013, 5, 11194-11206.

50 Y. Yan, H. Chen, H. Zhang, C. Guo, K. Yang, K. Chen, R. Cheng, N. Qian, N. Sandler, Y. S. Zhang, H. Shen, J. Qi, W. Cui and L. Deng, Biomaterials, 2019, 190-191, 97-110.

51 M. N. MacGregor-Ramiasa and K. Vasilev, Adv. Mater. Interfaces, 2017, 4, 1700381.

52 D. C. Trimbach, B. Keller, R. Bhat, S. Zankovych, R. Pöhlmann, S. Schröter, J. Bossert and K. D. Jandt, Adv. Funct. Mater., 2008, 18, 1723-1731.

53 R. M. Visalakshan and M. N. MacGregor, ACS Appl. Mater. Interfaces, 2019, 11, 27615-27623.

54 A. L. Sisson, D. Ekinci and A. Lendlein, Polymer, 2013, 54, 4333-4350.

55 M. R. Tavares, L. R. D. Menezes, J. C. Dutra Filho, L. M. Cabral and M. I. B. Tavares, Polym. Test., 2017, 60, 39-48.
56 H.-B. Chen, Y. Zhao, P. Shen, J.-S. Wang, W. Huang and D. A. Schiraldi, ACS Appl. Mater. Interfaces, 2015, 7, 20208-20214.

57 S. Maietta, T. Russo, R. De Santis, D. Ronca, F. Riccardi, M. Catauro, M. Martorelli and A. Gloria, Materials, 2018, 11, 312 .

58 S. DeLeon, A. Clinton, H. Fowler, J. Everett, A. R. Horswill and K. P. Rumbaugh, Infect. Immun., 2014, 82, 4718-4728.

59 R. Serra, R. Grande, L. Butrico, A. Rossi, U. F. Settimio, B. Caroleo, B. Amato, L. Gallelli and S. de Franciscis, Expert Rev. Anti-Infect. Ther., 2015, 13, 605-613.

60 L. Zhang, L. Wu, Y. Si and K. Shu, PLoS One, 2018, 13, e0209020.

61 C. F. Guerrero-Juarez, P. H. Dedhia, S. Jin, R. Ruiz-Vega, D. Ma, Y. Liu, K. Yamaga, O. Shestova, D. L. Gay, Z. Yang, K. Kessenbrock, Q. Nie, W. S. Pear, G. Cotsarelis and M. V. Plikus, Nat. Commun., 2019, 10, 650.

62 Z. Li, R. Yang, M. Yu, F. Bai, C. Li and Z. L. Wang, J. Phys. Chem., 2008, 112, 20114-20117.

63 N. Ninan, N. Goswami and K. Vasilev, Nanomaterials, 2020, 10, 967.

64 N. Strbo, N. Yin and O. Stojadinovic, Adv. Wound Care, 2014, 3, 492-501.

65 S. Ellis, E. J. Lin and D. Tartar, Curr. Dermatol. Rep., 2018, 7, 350-358.

66 S. Guo and L. A. Dipietro, J. Dent. Res., 2010, 89, 219-229.

67 M. Ritsu, K. Kawakami, E. Kanno, H. Tanno, K. Ishii, Y. Imai, R. Maruyama and M. Tachi, J. Dermatol. Surg., 2017, 21, 14-19.

68 C. You, Q. Li, X. Wang, P. Wu, J. K. Ho, R. Jin, L. Zhang, H. Shao and C. Han, Sci. Rep., 2017, 7, 10489.

69 M. E. Samberg, S. J. Oldenburg and N. A. Monteiro-Riviere, Environ. Health Perspect., 2010, 118, 407-413. 